\title{
STATE-OF-THE-ART OF THE PHYSiCAL LAYER IN UNDERWATER WIRELESS SENSOR NETWORKS
}

\author{
Faiza Al-Salti ${ }^{1}$ and N. Alzeidi ${ }^{2}$ \\ ${ }^{1}$ Department of Computing, Muscat College, Oman \\ ${ }^{2}$ Department of Computer Science, Sultan Qaboos University, Oman
}

\begin{abstract}
With the current technology revolution, underwater wireless sensor networks (UWSNs) find several applications such as disaster prevention, water quality monitoring, military surveillance and fish farming. Nevertheless, this kind of networks faces a number of challenges induced by the nature of the underwater environment and its influence on the network physical media. Therefore, the ultimate objective of this paper is to lay down the key aspects of the physical layer of the underwater sensor networks (UWSNs). It discusses issues related to the characteristics and challenges of the underwater communication channel, differences between terrestrial wireless sensor networks and UWSNs, and acoustic propagation models in underwater. The paper also surveys some of the underwater acoustic modems. This study is essential to better understand the challenges of designing UWSNs and alleviate their effects.
\end{abstract}

\section{KEYWORDS}

Underwater wireless sensor networks, physical layer, acoustic, communication, channel models \& acoustic modem

\section{INTRODUCTION}

The oceans, seas, rivers and lakes cover around $75 \%$ of the Earth's surface, and with the increasing importance of these areas in human life, there is a strong demand to investigate the unexplored regions and make use of their valuable treasures. Underwater Sensor Networks (UWSNs) are considered a promising candidate for achieving this objective.

This kind of network consists of a collection of sensors deployed underwater to perform a collaborative task. Sensors can measure a variety of parameters and conditions such as temperature, salinity, pressure, noise level, and nutrient concentration [1]. They can also detect and track the presence or absence of certain types of objects [2]. Therefore, UWSNs enable various applications including scientific, military and commercial applications. Commercial applications of UWSNs are typically associated with monitoring and controlling commercial activities in underwater environment such as monitoring of underwater pipelines [3], fish farming [4] and deep-water oil drilling [5]. Scientific applications are mainly associated with applications responsible for monitoring and observing the environment for scientific research objectives. Examples of these applications are disaster detection and early warnings [4], water quality monitoring [6], coral reef monitoring [7] and red tides monitoring [8]. Perhaps the first underwater acoustic network developed belongs to military applications. It was developed during the cold war to monitor the movement of soviet submarines [4]. Military applications focuses mainly on applications such as military surveillance and reconnaissance [9], intruder detections 
and mine detections [10]. It is worth mentioning that The Office of Naval Research supports a number of programs in acoustic communication [11].Understanding the physical medium upon which the communication is carried on and the characteristics of the employed signal to transmit data is essential in designing the network effectively and achieving a good performance. This is due to their great influence on the development of the higher layers' protocols. For example, a proper selection of the frequency and the transmission distance affect the overall available bandwidth and data rate. In addition, some of the MAC protocols that require exchanging control packets before actual data transfer may not be suitable for UWSNs due to the high propagation delay. Moreover, since energy is a critical resource, power control might be necessary and welldesigned routing protocols can help in preserving the resources. Therefore, this paper aims to lay down the fundamental basis of the physical layer of UWSNs and to survey the state-of-the-art of this layer.

The rest of the paper is organized as follows. Section 2 discusses the characteristics and challenges of the communication channel in underwater networks. Section 3 presents the differences between terrestrial wireless sensor networks and UWSNs. Some of the existing acoustic propagation models in underwater are discussed in section 4 . This is followed by a discussion on the selection of the transmission range in section 5. Section 6 surveys some of the existing commercial acoustic modems and their capabilities. Conclusion and some open research directions are presented in section 7.

\section{Characteristics of The Communication Channel}

Acoustic waves are the only feasible physical layer technology for underwater networks communication [12] [13]. In fact, electromagnetic waves propagate for a very short range (less than 1 meter at $1 \mathrm{MHz}$ [13]) in underwater due to the high attenuation and absorption at high frequency. In seawater, the absorption of an electromagnetic signal is approximately $(45 \sqrt{f}) \mathrm{dB} / \mathrm{km}$, where $\mathrm{f}$ is the frequency in hertz[14]. This is three orders of magnitude higher than the absorption of the acoustic signal in water. Although an electromagnetic wave can propagate for a reasonable distance at low frequency, it requires high transmission power and large antenna. Optical links are also not good for use in water for many reasons. First, the absorption of the optical signal in water is very high and hence can propagate only for short distances (less than 10 meters at $1 \mathrm{GHz}[13]$ ). Second, it suffers from scattering[15]. Third, it needs a precise positioning for narrow beam optical transmitter, which is hard to provide in underwater environments[12][15].

Although acoustic communication is the only suitable medium in underwater environment, it is considered as one of the most tough communication media in use today[16]. This is due to the fact that acoustic signals are affected by many factors, which pose several challenges and intricacies in designing UWSNs[15][17]. These factors include:

- Multipath: Due to the reflection (at the surface, at the bottom or on any object in water) and refraction (in water) of the acoustic wave, duplicate copies of the signal may reach the destination. The spreading of the same signal through multiple paths can cause inter-symbol interference (ISI) as signals may arrive in an overlapping time interval. The reflection strongly depends on both the depth and the distance between the source and the destination[17]. For example, the reflection of the sound wave at the surface and at the bottom in shallow water (i.e. water with depth less than $100 \mathrm{~m}[15]$ ) dominates the cause of the multipath phenomenon because the transmission range of the signal is larger than the water depth. In deep water, on the other hand, the reflection at the surface and at the bottom can be neglected but, the variation in the sound speed results in multipath propagation of the signal. 
- Path loss: It consists of:

- Absorptive loss: During propagation, the energy of the signal may be converted into heat due to the inelasticity of the medium. According to[18], the absorptive loss of an acoustic signal can be expressed as $\sqrt{\alpha(f)^{d}}$ where $\mathrm{d}$ is the distance (in $\mathrm{Km}$ ) between the transmitter and the receiver and $\overline{\alpha(f)}$ is the absorption coefficient (in $\mathrm{dB} / \mathrm{Km}$ ) at frequency $\mathrm{f}$ (in $\mathrm{kHz}$ ), and it can be calculated using the Thorp's formula as given in (1).

$$
\alpha(f)=0.11 \frac{f^{2}}{1+f^{2}}+44 \frac{f^{2}}{4100+f^{2}}+0.000275 f^{2}+0.003
$$

Figure 1 shows that the absorption coefficient increases rapidly with the increase in the frequency, and the overall absorptive loss increases substantially with the increase in the distance and frequency as demonstrated in Figure 2.

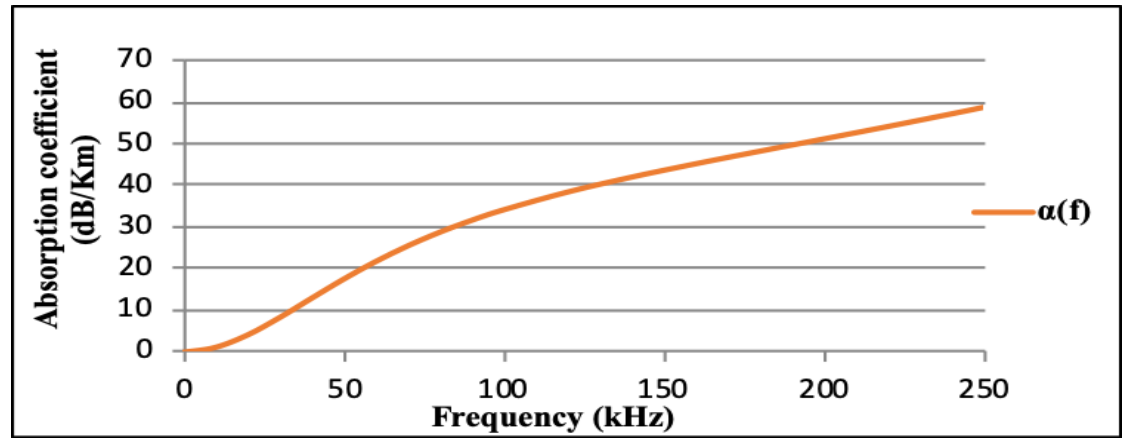

Figure 1: The relation between frequency and absorption coefficient

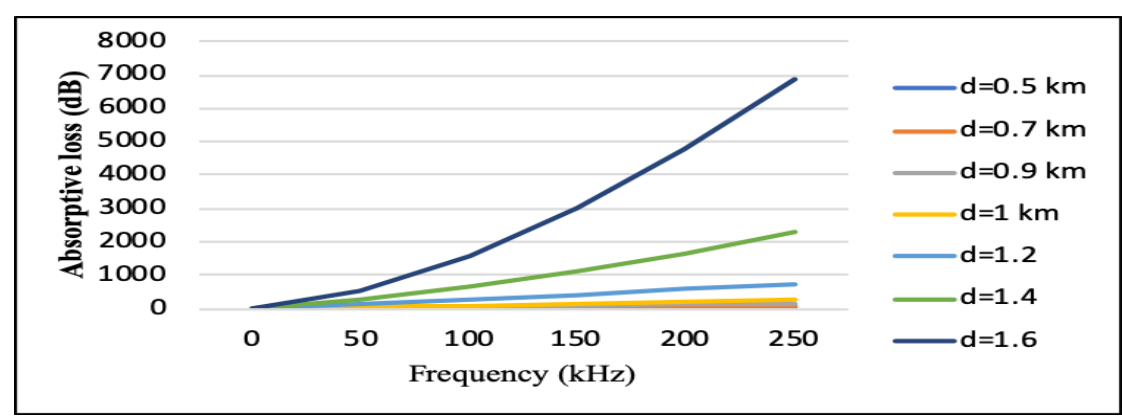

Figure2: Absorptive loss under different values of frequency and distances

- Geometric spreading: due to the expansion of the wave as it propagates toward the receiver, the wave loses its energy. Geometric spreading is frequency-independent. It mainly depends on the propagation distance. Geometric spreading can be classified into spherical and cylindrical. The power loss caused by spherical spreading which characterizes the deep-water communication is directly proportional to the square of the distance. On the other hand, the cylindrical spreading which characterizes the shallow water communication is directly proportional to the distance[15][17].

- Scattering: scattering is a physical phenomenon that occurs when a particle collides with another particle causing it to change its trajectory as demonstrated in Figure 
Surface scattering causes the signal to lose its power. In addition, scattering causes spreading in delay of each surface bounced path, which lowers the spatial correlation of scattered signals[17][19]. This results in multipath phenomenon discussed above. Section 4 summarizes some of the existing models for path loss of an acoustic signal.

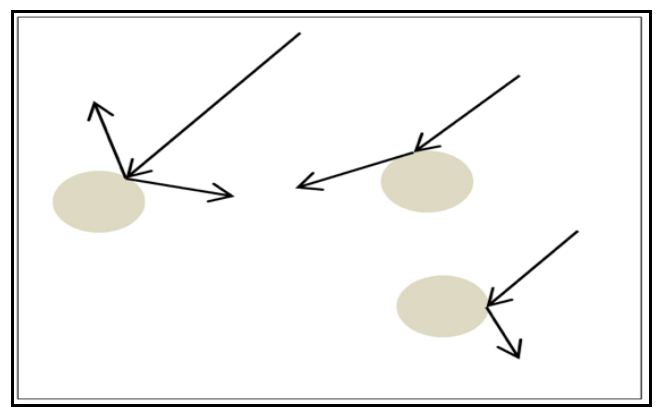

Figure 1: Illustration of the scattering phenomenon

- Ambient noise: The ambient noise $(\mathrm{N})$ can be modelled as a result of four different sources namely the turbulence $(\mathrm{Nt})$, shipping $(\mathrm{Ns})$, waves $(\mathrm{Nw})$ and thermal $(\mathrm{Nth})$ noise [20][21] as follows:

$$
\begin{aligned}
& 10 \log \mathrm{N}_{\mathrm{t}}(\mathrm{f})=17-30 \log f \\
& 10 \log \mathrm{N}_{\mathrm{s}}(\mathrm{f})=40-20(\mathrm{~s}-0.5)+26 \log f-60 \log (\mathrm{f}+0.03) \\
& 10 \log N_{\mathrm{w}}(\mathrm{f})=50+7.5 \mathrm{w}^{1 / 2}+20 \log f-40 \log (\mathrm{f}+0.4) \\
& 10 \log \mathrm{N}_{\mathrm{th}}(\mathrm{f})=-15+20 \log f \\
& N(f)=N_{t}(f)+N_{s}(f)+N_{w}(f)+N_{t h}(f)
\end{aligned}
$$

Where s expresses the amount of naval traffic near the network area and it ranges from 0 to 1 inclusive (i.e. 0 means no shipping and 1 means a very shipping route) and $\mathrm{w}$ is the wind speed in $\mathrm{m} / \mathrm{s}$. Clearly, the level of noise is frequency-dependent. The higher is the frequency of the signal, the higher the error rate of the acoustic channel.

- High and variable propagation delay: The speed of an acoustic signal in water ranges from 1450 to $1540 \mathrm{~m} / \mathrm{s}[22]$. A typical acoustic signal speed is about $1500 \mathrm{~m} / \mathrm{s}$ and depends on water properties including temperature, salinity and depth. The following equation is a general formula for calculating the speed of sound $v$ in sea water and it is known as Mackenzie equation[23]:

$$
\begin{aligned}
& v=1448.96+4.591 T-5.304 * 10^{-2} T^{2}+2.374 * 10^{-4} T^{3}+ \\
& 1.340(S-35)+1.630 * 10^{-2} D+1.675 * 10^{-7} D^{2}-1.025 * \\
& 10^{-2} T(S-35)-7.139 * 10^{-13} T D^{3}
\end{aligned}
$$

Where $\mathrm{v}$ is the speed of sound in $\mathrm{m} / \mathrm{s}$, $\mathrm{T}$ is the temperature in degrees Celsius, $\mathrm{S}$ is the salinity in parts per thousands and D is the depth in meters. The equation is valid for temperatures between 2 to $30 \mathrm{Co}$, salinity between 25 to 40 parts per thousand and depth in the range 0 to $8000 \mathrm{~m}$.

The typical speed is more than four times the speed of sound in air, but five orders of magnitude less than the speed of light $(3 * 108 \mathrm{~m} / \mathrm{s})$ in air. The slow speed of sound in water causes high propagation delay of the acoustic signal which limits the throughput of 
the network. In addition, the channel also suffers from variable propagation delay and its effect can be harmful in designing an efficient network. For example, it might be difficult to estimate the round-trip time accurately which is an important parameter in some protocols. The speed of the acoustic signal increases by $4.0 \mathrm{~m} / \mathrm{s}$ when the temperature increases by $1 \mathrm{oC}$. When the salinity of the water increases by 1 practical salinity unit (PSU), the speed increases by $1.4 \mathrm{~m} / \mathrm{s}$. By increasing the depth under water by $1 \mathrm{~km}$, the speed of sound increases by $17 \mathrm{~m} / \mathrm{s}$ [14].

- Doppler shift and spread: Doppler spread causes the performance of acoustic channels to degrade in high data transmissions[17]. Particularly, it causes adjacent symbols of the received signals to interfere with each other. Doppler shift of the acoustic signal in water is relatively high due to the low carrier frequency. Particularly, the ratio of the Doppler shift to carrier frequency is in the range 10-3 - 10-4 compared to 10-7 - 10-9 for terrestrial RF channel[12].

The aforementioned factors make underwater acoustic channels temporally and spatially variable, and also make the bandwidth of the channel limited and dependent on both communication range and frequency. Specifically, less bandwidth is achieved when the communication range increases. Thus, it is worth mentioning that network developers and designers should consider the relation between the transmission range and the available bandwidth (and hence, the data rate) when designing a network in order to achieve good performance. Section 5 surveys some studies regarding this issue. In addition, multi-path, path-loss and Doppler spread cause high bit error rate and delay variance and need to be carefully addressed when designing acoustic modems and higher layers' protocols.

\section{DIFFERENCES BETWEEN TERRESTRIAL WSNS AND UWSNS}

Underwater wireless sensor networks (UWSNs) are different from terrestrial wireless sensor networks (WSNs) due to the different characteristics of the transmission medium and to the type of signal used for communication. The most important differences are described below and summarized in Table 1.

- Propagation delay: As mentioned in Section 2, the typical acoustic signal speed underwater is $1500 \mathrm{~m} / \mathrm{s}$ which is five orders of magnitude lower than the speed of radio wave $\left(3 * 10^{8} \mathrm{~m} / \mathrm{s}\right)$ used in terrestrial WSNs. This slow speed leads to high propagation delay (in the order of hundreds of milliseconds) of an acoustic signal and when combined with the low bandwidth of the channel, it results in large end-to-end delay, and thus, affects the performance of applications especially those that pose constraints on the communication time. In addition, the slow speed induces more challenges in the localization process of sensor nodes. For example, when a node tries to send its new location to other nodes, the message may arrive at a time the information is no longer useful (e.g. the node has moved to another point), and hence, results in incorrectly updating the node's location or wrong location tags of the sensed data.

- Node mobility: In most terrestrial WSNs, sensor nodes are generally assumed to be fixed, and hence, different topologies can be used. On the other hand, underwater sensor nodes are assumed to move with water currents (e.g. in typical underwater conditions, underwater objects can move at speed 3-6 km/h[24]). Therefore, higher layers' protocols need to consider the mobility of the sensor nodes in order to achieve acceptable performance.

- Available bandwidth: Terrestrial WSNs usually have a high bandwidth in the order of $\mathrm{MHz}[14]$. However, underwater acoustic channels have a limited bandwidth that highly 
depends on the transmission range and frequency. For example, the bandwidth for shortrange UWSN operating over several tens of meters is a hundred of $\mathrm{kHz}$; while for longrange UWSN, the bandwidth is limited to a few $\mathrm{kHz}$ [14] [24][25].

- Cost: Underwater network devices are more expensive in manufacturing, deployment, maintenance and recovery than those used in terrestrial WSNs. An acoustic modem with a rugged housing, for instance, costs around \$3000[12]. Moreover, underwater sensor

o nodes require hardware protection against fouling and corrosion. The deployment can also be expensive, and it depends on network size and weather in the targeted environment. For example, an oceanographic research vessel costs $\$ 5000$ - $\$ 25000$ per day[12].

- Deployment: While dense deployment characterizes terrestrial WSNs, UWSNs tend to be sparse due to the high cost and to the vast volume that needs to be covered. Moreover, the deployment in terrestrial WSNs is deterministic since nodes are deployed manually in the environment. On the contrary, the common deployment strategy in UWSNs is a nonuniform and random deployment.

- Energy Consumption: Energy is a primary concern in UWSNs. Underwater acoustic communication consumes more energy than terrestrial radio communication. This is due to the longer distances between nodes (i.e. results from sparse deployment) and to the more complex signal processing in UWSNs. Moreover, batteries for terrestrial sensor nodes can be easily recharged and replaced. However, this is not the case for the batteries of underwater sensor nodes which tend to have limited power and cannot be easily replaced or recharged. In addition, solar energy cannot be exploited in underwater environment. Different approaches have been proposed to enable underwater equipment to save their energy for longer time. Special routing algorithms (e.g. PER[26]) and MAC protocols (e.g. the MAC protocol proposed in[27]) are designed primarily for this purpose. It is also worth mentioning that there is a significant difference between the power required to transmit an acoustic signal and the power required to receive or listen to an acoustic signal. The ratio of power required for transmission to the power required for reception is 125:1[28]. However, the power required to transmit or receive a radio signal in terrestrial WSNs is almost the same.

- Reliability: The bit-error rate for communication channels in UWSNs is much higher than in terrestrial WSNs. In addition, underwater sensor nodes may lose their connectivity for several reasons (e.g. mobility), which produces holes in the network (i.e. areas where there are no nodes to relay data to the next hop).

- Spatial Correlation: Due to the high cost of underwater sensor nodes compared to the terrestrial sensor nodes, distances between deployed sensors in UWSNs can be large. This cause readings from underwater sensor nodes to be un-correlated and the longer the distance, the higher the distortion of acoustic signals. In contrast, readings from sensor nodes in WSNs are correlated.

- Localization: Localization is the process of identifying the nodes' locations in sensor networks[29]. In terrestrial WSNs, localization is performed using GPS service. However, radio waves are highly attenuated in UWSNs, hence GPS cannot be used for localizing underwater nodes. Therefore, UWSNs should rely on localization schemes that do not depend on GPS signals.

- Lack of standards: Standards for terrestrial WSNs are well established and internationally accepted and because of the unique features of the UWSNs, these standards cannot be applied for such networks. UWSNs lacks such standards and much more work needs to be done in this field[15]. 
Table 1: Comparison between terrestrial WSNs and UWSNs

\begin{tabular}{|c|c|c|}
\hline & Terrestrial WSNs & UWSNs \\
\hline Propagation speed & $\operatorname{High}\left(3 * 10^{8} \mathrm{~m} / \mathrm{s}\right)$ & Very low $(1500 \mathrm{~m} / \mathrm{s})$ \\
\hline Propagation delay & Short $(\mu \mathrm{s})$ & Long $(\mathrm{ms})$ \\
\hline Available bandwidth & $\begin{array}{l}\text { High }(\mathrm{MHz}) \& \text { independent of } \\
\text { distance }\end{array}$ & $\begin{array}{c}\text { Low }(\mathrm{KHz}) \text { and depends on } \\
\text { distance }\end{array}$ \\
\hline Noise & White noise & Depends on frequency \\
\hline Devices & Cheaper & $\begin{array}{l}\text { Expensive \& require hardware } \\
\text { protection }\end{array}$ \\
\hline Energy cost & $\begin{aligned} \text { Transmit }(\mathrm{Tx}) & \sim \text { receive }(\mathrm{Rx}) \sim \text { idle } \\
& >\text { sleep }\end{aligned}$ & $\begin{array}{c}\text { Transmit }(\mathrm{Tx})>>\text { receive }(\mathrm{Rx})> \\
\text { idle }>>\text { sleep } \\
\end{array}$ \\
\hline Error rate & Low & high \\
\hline Mobility & Almost negligible & $3-6 \mathrm{~km} / \mathrm{h}$ \\
\hline Normal deployment & Dense & Sparse \\
\hline localization & Via GPS & GPS-free schemes \\
\hline Available standards & Internationally accepted standards & No standards \\
\hline
\end{tabular}

\section{Acoustic Propagation Model}

This section summarizes two of the well-known underwater acoustic propagation models. The obtained results of these models may be used as a guide to develop underwater acoustic modems and to describe the expected sound profile for various underwater environments[30]. Yet, there is no standard underwater acoustic propagation model.

\section{- Urick description and Thorp formula}

The simplest model for the transmission loss of underwater acoustic signal depends on the frequency and the transmission distance. The attenuation of the signal can be represented as follows[31]:

$$
\begin{aligned}
& A(d, f)=d^{k} a^{d} \\
& a=10^{\alpha(f) / 10}
\end{aligned}
$$

where $d$ is the distance (in $\mathrm{Km}$ ) that the signal travels, and $k$ is the spreading factor (i.e. $k$ is 1 for cylindrical, 2 for spherical and 1.5 for practical spreading), $f$ is the frequency in $\mathrm{KHz}$, and $\alpha(f)$ is the absorption coefficient in $\mathrm{dB} / \mathrm{Km}$ and calculated using the Thorp's formula as given in (1).

In Figure 4 we plot the effect of the frequency on the attenuation for different distances travelled by the signal. Each line is related to a specific distance. Again, the attenuation increases rapidly with the increase in the frequency and distance. Note that the geometric practical spreading $(\mathrm{K}=1.5)$ is used to draw the figure; though, using any of the other two spreading (i.e. spherical or cylindrical) show similar trends. 


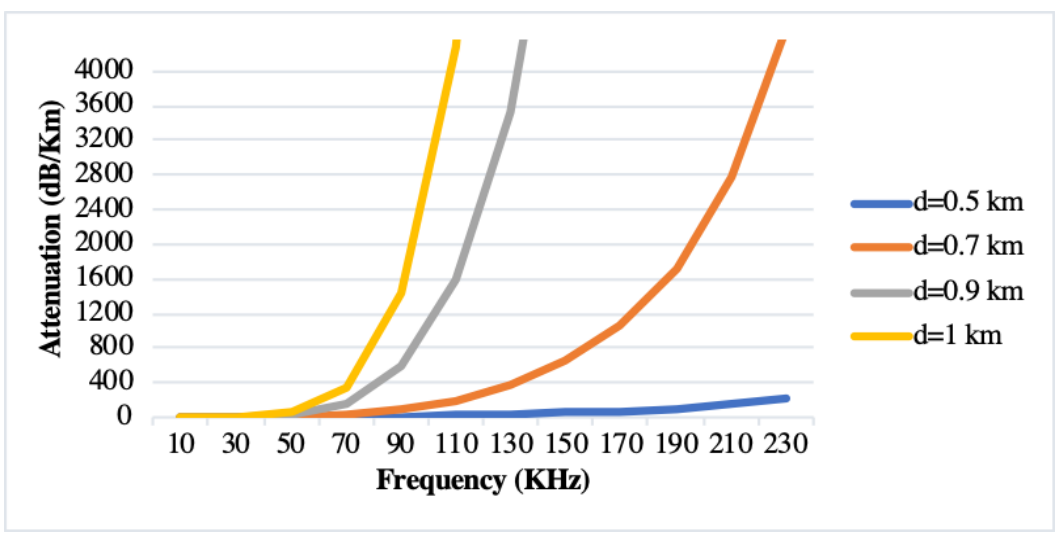

Figure 4: The relation between frequency and attenuation for different distances

Although the model is simple, it doesn't consider the effect of the environmental parameters such as the depth of the sender and receiver of the signal. In other word, it deals with nodes as if they are at the same depth. For example, the propagation loss for a signal received by a node at distance 500 meters and at depth 20 meters is the same as the propagation loss for the signal with the same distance but located at depth 400 meters.

\section{- Monterey-Miami Parabolic Equation (MMPE) Model}

The transmission loss defined in the previous model is simple and not accurate since it depends only on frequency and distance. The Monterey-Miami Parabolic Equation (MMPE) Model developed by Kevin Smith and Frederick Tappert [32] provided more accurate prediction of the underwater acoustic propagation loss. It is a parabolic equation model and is based on the split-step Fourier algorithm. It considers the effects of several factors such as surface activity, depth of the nodes and salinity changes. Although this model is more accurate than the above model, it requires intensive computations. Therefore a large amount of time is required to obtain the propagation loss[22][30]. That is not feasible for simulation tools and it might not be feasible also to be programmed in actual sensor nodes due to their constrained computational resources. The study in [33] provides an approximation to the MMPE model. The approximated formula to the propagation loss is as follows:

$$
P L(t)=m\left(d_{A}, d_{B}, s, f\right)+w(t)+e(s)
$$

Where $\overline{P L(t)}$ is the propagation loss while transmitting from node $A$ to node $B$, $m\left(d_{A}, d_{B}, s, f\right)$ is the propagation loss without random and periodic components and it is resulted from the regression of MMPE data. $d_{A}$ and $d_{B}$ are the depth of the sender and receiver, respectively (in meters). $s$ is the distance between $A$ and $B$ and it is calculated as $\sqrt{\left(d_{A}-d_{B}\right)^{2}+r^{2}}$ where $r$ is the horizontal distance (in meters) between $A$ and $B$ and it is called the range in the MMPE Model. $f$ is the frequency in $\mathrm{kHz}$ of the transmitted acoustic signal. $w(t)$ is the periodic function to estimate the signal loss due to the wave motion. $e(s)$ is a random term used to estimate the effects of the noise. For more details about the MMPE model and its approximation model, refer to [32] and[33], respectively. 


\section{ON THE SElECtion OF Transmission RANGe}

The transmission range $R$ of a node can be used to determine the appropriate transmission power as follows[34]:

$$
P=S N R \cdot A(R, f) \cdot N(f) \cdot B \quad(7)
$$

Where $S N R$ is the signal to noise ratio, $A(R, f)$ is the attenuation as given in (4), $N(f)$ is the noise level and $B$ is the bandwidth.

Porto et al. [34] conducted a simulation study to investigate the effect of transmission power on the energy consumption and the throughput. They concluded that the optimal transmission power that maximizes the energy efficiency is the one that ensures a minimal connectivity between nodes (i.e. ensures connecting a node to another node closer to the sink than the node itself). They also concluded that this transmission power also results in maximizing the overall throughput. Therefore, in order to improve the performance, the authors suggested that nodes may be equipped with a service to control the transmission power to ensure a minimal connectivity based on their positions but at the expense of incurring extra cost.

The authors in [35] investigated the effect of the selected transmission range on the energy efficiency and the network connectivity of the UWSNs. They provided an analytical model showing the trade-off between energy efficiency and network connectivity based on the selected transmission range in a random network. Having larger transmission range increases the network connectivity, but at the cost of increasing the energy consumption. On the other hand, reducing the transmission range saves the energy; however, it might not lead to a satisfactory connectivity (i.e. connectivity between nodes might be lost). Network connectivity might be increased by increasing either the transmission range which costs extra energy and interference or by increasing the node density which incurs hardware cost. Therefore, network designers should determine the trade-off and select appropriate parameters for the network setup.

\section{Acoustic Modems and Their Capabilities}

The acoustic modems can be classified as commercial acoustic modems and research acoustic modems. Tables 2 summarizes the characteristics of some of the commercial acoustic modems developed for UWSNs, respectively. 
International Journal of Wireless \& Mobile Networks (IJWMN) Vol. 11, No. 6, December 2019

Table 2: Characteristics of commercial acoustic modems for UWSNs

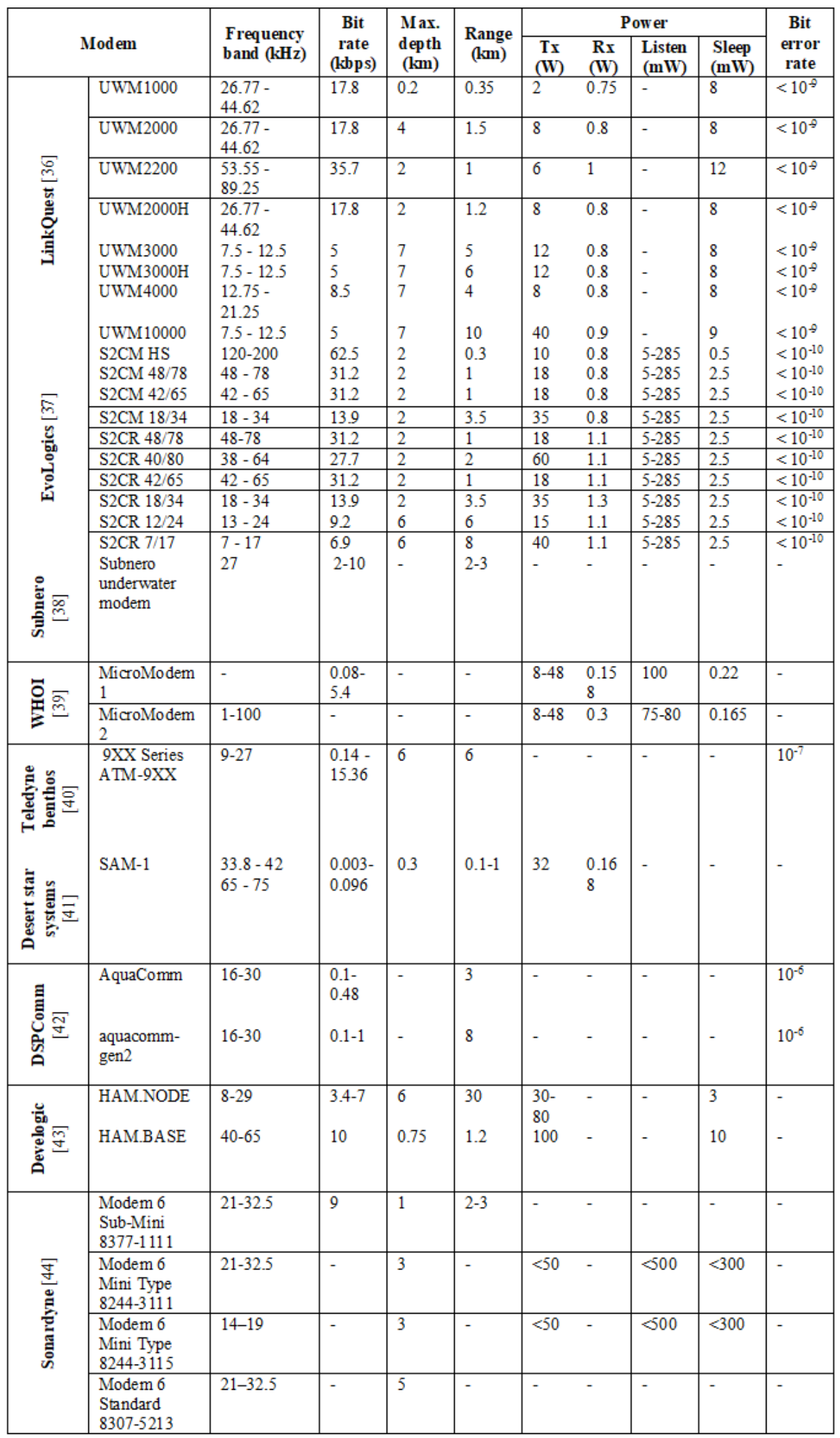




\section{CONCLUSION AND FUTURE RESEARCH DiRECTIONS}

This paper presented a comprehensive description of the state-of-the-art related to physical layer in UWSNs. It discussed in brief the difference between the possible communication channels underwater, their characteristics and challenges of using them. After that, the differences between the terrestrial WSNs and UWSNs are presented. This is followed by presenting some of the modelling approaches for underwater acoustic propagation and existing commercial acoustic modems. The paper also discussed how to select appropriate transmission range to gain better performance. Finally, the paper investigated the existing commercial acoustic modems and compared between them.

For future research, there is a demand for minimizing the bit error rate by for example developing algorithms for Doppler correction. In addition, there is still a need for developing inexpensive acoustic modems which will expand the use of UWSNs and facilitate further applications.

\section{ACKNOWLEDGMENT}

This work is supported by The Research Council (TRC) of the Sultanate of Oman under the research grant number RC/SCI/COMP/15/02.

\section{REFERENCES}

[1] I. F. Akyildiz, W. Su, Y. Sankarasubramaniam, and E. Cayirci, "Wireless sensor networks: a survey," Computer Networks, vol. 38, no. 4, pp. 393-422, March 2002.

[2] G. Isbitiren and O. B. Akan, "Three-Dimensional Underwater Target Tracking With Acoustic Sensor Networks," IEEE Transactions on Vehicular Technology, vol. 60, no. 8, pp. 3897-3906, October 2011.

[3] N. Mohamed, I. Jawhar, J. Al-Jaroodi, and L. Zhang, "Sensor network architectures for monitoring underwater pipelines,” Sensors (Basel)., vol. 11, no. 11, pp. 10738-64, January 2011.

[4] S. Q. Mohsin Murad, Adil A. Sheikh, Muhammad Asif Manzoor, Emad Felemban, "A Survey on Current Underwater Acoustic Sensor Network Applications,” Int. J. Comput. Theory Eng., vol. 7, no. 1, pp. 51-56, 2015.

[5] F. J. L. Ribeiro, A. de Castro Pinto Pedroza, and L. H. M. K. Costa, "Underwater monitoring system for oil exploration using acoustic sensor networks,” Telecommun. Syst., vol. 58, no. 1, pp. 91-106, Dec. 2014.

[6] G. Xu, W. Shen, and X. Wang, "Applications of wireless sensor networks in marine environment monitoring: a survey.," Sensors (Basel)., vol. 14, no. 9, pp. 16932-54, January 2014.

[7] M. N. Jaroensutasinee, M., K. Jaroensutasinee, S. Bainbridge, T. Fountain, S. Chumkiew, P. Noonsang, U. Kuhapong, S. Vannarat, A. Poya, "Sensor Networks Applications for Reefs at Racha Island, Thailand," in Proceedings of the 12 th International Coral Reef Symposium, 9-13 July 2012, Cairns, Australia.

[8] R. Kastner, A. Lin, C. Schurgers, J. Jaffe, P. Franks, and B. S. Stewart, "Sensor platforms for multimodal underwater monitoring," in 2012 International Green Computing Conference (IGCC), 4-8 June 2012, pp. 1-7, San Jose, CA, USA.

[9] E. Cayirci, H. Tezcan, Y. Dogan, and V. Coskun, "Wireless sensor networks for underwater survelliance systems," Ad Hoc Networks, vol. 4, no. 4, pp. 431-446, July 2006.

[10] L. Freitag, M. Grund, C. Von Alt, R. Stokey, and T. Austin, "A Shallow Water Acoustic Network for Mine Countermeasures Operations with Autonomous Underwater Vehicles," Underwater Defense Technology (UDT), pp. 1-6, June 2005.

[11] R. Headrick and L. Freitag, "Growth of underwater communication technology in the U.S. Navy," IEEE Communications Magazine, vol. 47, no. 1, pp. 80-82, January 2009.

[12] Z. Jiang, "Underwater Acoustic Networks - Issues and Solutions," International Journal of Intelligent Control and Systems, vol. 13, no. 3, pp. 152-161, September 2008.

[13] U. M. Qureshi, F. K. Shaikh, Z. Aziz, S. M. Z. S. Shah, A. A. Sheikh, E. Felemban, and S. Bin Qaisar, "RF Path and Absorption Loss Estimation for Underwater Wireless Sensor Networks in 
Different Water Environments.," Sensors (Basel)., vol. 16, no. 6, June 2016.

[14] M. Ayaz, I. Baig, A. Abdullah, and I. Faye, "A survey on routing techniques in underwater wireless sensor networks," Journal of Network and Computer Applications, vol. 34, no. 6, pp. 1908-1927, November 2011.

[15] I. F. Akyildiz, D. Pompili, and T. Melodia, "Underwater acoustic sensor networks: research challenges," Ad Hoc Networks, vol. 3, no. 3, pp. 257-279, May 2005.

[16] M. Stojanovic and J. Preisig, "Underwater acoustic communication channels: Propagation models and statistical characterization," IEEE Communication Magazine, vol. 47, no. 1, pp. 84-89, January 2009.

[17] L. Lanbo, Z. Shengli, and C. Jun-Hong, "Prospects and problems of wireless communication for underwater sensor networks," Wireless Communications and Mobile Computing, vol. 8, no. 8, pp. 977-994, October 2008.

[18] S. Climent, A. Sanchez, J. V. Capella, N. Meratnia, and J. J. Serrano, "Underwater acoustic wireless sensor networks: advances and future trends in physical, MAC and routing layers," Sensors (Basel)., vol. 14, no. 1, pp. 795-833, January 2014.

[19] J. Preisig, "Acoustic propagation considerations for underwater acoustic communications network development," ACM SIGMOBILE Mobile Computing and Communications Review, vol. 11, no. 4, pp. 2-10, October 2007.

[20] M. Stojanovic, "On the relationship between capacity and distance in an underwater acoustic communication channel," ACM SIGMOBILE Mobile Computing and Communications Review, vol. 11, no. 4, pp. 34-43, October 2007.

[21] P. Casari and M. Zorzi, "Protocol design issues in underwater acoustic networks," Computer Communications, vol. 34, no. 17, pp. 2013-2025, November 2011.

[22] J. Llor, E. Torres, P. Garrido, and M. P. Malumbres, "Analyzing the behavior of acoustic link models in underwater wireless sensor networks," in Proceedings of the 4th ACM workshop on Performance monitoring and measurement of heterogeneous wireless and wired networks PM2HW2N '09, 2009, 26 October 2009, pp. 9-16, New York, USA.

[23] J.-H. Cui, J. Kong, M. Gerla, and S. Zhou, "The challenges of building mobile underwater wireless networks for aquatic applications," IEEE Networks, vol. 20, no. 3, pp. 12-18, May 2006.

[24] K. V. Mackenzie, "Nine-term equation for sound speed in the oceans," The Journal of the Acoustical Society of America, vol. 70, no. 3, p. 807, September 1981.

[25] J.-H. Cui, J. Kong, M. Gerla, and S. Zhou, "The challenges of building mobile underwater wireless networks for aquatic applications," IEEE Networks, vol. 20, no. 3, pp. 12-18, May 2006.

[26] N. Chirdchoo, W.-S. Soh, and K. C. Chua, "Sector-Based Routing with Destination Location Prediction for Underwater Mobile Networks," in Proceedings of International Conference on Advanced Information Networking and Applications Workshops, 26-29 May 2009, pp. 1148-1153, Bradford, England.

[27] C.-J. Huang, Y.-W. Wang, H.-H. Liao, C.-F. Lin, K.-W. Hu, and T.-Y. Chang, "A power-efficient routing protocol for underwater wireless sensor networks," Applied Soft Computing, vol. 11, no. 2, pp. 2348-2355, March 2011.

[28] V. Rodoplu and M. K. Park, “An Energy-Efficient MAC Protocol for Underwater Wireless Acoustic Networks," in Proceedings of OCEANS 2005 MTS/IEEE, 7-23 September 2005, pp. 1198-1203, Washington, DC, USA.

[29] K. Chen, M. Ma, E. Cheng, F. Yuan, and W. Su, "A Survey on MAC Protocols for Underwater Wireless Sensor Networks," IEEE Communincations Surveys \& Tutorials, vol. 16, no. 3, pp. 14331447, Third Quarter 2014.

[30] V. Chandrasekhar, W. K. Seah, Y. S. Choo, and H. V. Ee, "Localization in underwater sensor networks: survey and challenges," in Proceedings of the 1st ACM international workshop on Underwater networks, WUWNet '06, 25 September 2006, pp. 33-40, Los Angeles, CA, USA.

[31] G. Xie, J. Gibson, and L. Diaz-Gonzalez, "Incorporating Realistic Acoustic Propagation Models in Simulation of Underwater Acoustic Networks: A Statistical Approach," in OCEANS 2006, 18-21 September 2006, pp. 1-9, Boston, MA, USA.

[32] L. M. Brekhovskikh and I. P. Lysanov, Fundamentals of Ocean Acoustics. Springer Science \& Business Media, March 2003.

[33] K. B. SMITH, "Convergence, Stability, and Variability of Shallow Water Acoustic Predictions using A Split-Step Fourier Parabolic Equation Model,” Journal of Computational Acoustics, vol. 09, no. 01, pp. 243-285, March 2001. 
[34] L. J. Solorzano, "Underwater Acoustic Networks: An Acoustic Propagation Model for Simulation of Underwater Acoustic Networks," NAVAL POSTGRADUATE SCHOOL, 2005.

[35] A. Porto and M. Stojanovic, "Optimizing the Transmission Range in an Underwater Acoustic Network," in Oceans 2007, 29 September - 4 October 2007, pp. 1-5, Vancouver, BC, Canada.

[36] M. Gao, C. H. Foh, and J. Cai, "On the selection of transmission range in underwater acoustic sensor networks.," Sensors, vol. 12, no. 4, pp. 4715-29, January 2012.

[37] "LinkQuest: Underwater acoustic modem models." [Online]. Available: http://www.linkquest.com/html/models1.htm. [Accessed: 10 April 2018].

[38] "EvoLogics: Underwater Acoustic Modems." [Online]. Available: https://www.evologics.de/en/products/acoustics/index.html. [Accessed: 02 September 2018].

[39] "SUBNERO: acoustic modem." [Online]. Available: https://subnero.com/technology/wnc/. [Accessed: 02 September 2018].

[40] E. Gallimore, J. Partan, I. Vaughn, S. Singh, J. Shusta, and L. Freitag, "The WHOI micromodem-2: A scalable system for acoustic communications and networking," in OCEANS 2010 MTS/IEEE SEATTLE, 20-23 September 2010, pp. 1-7, Seattle, WA, USA.

[41] "Teledyne Benthos Acoustic Modems." [Online]. Available: http://teledynebenthos.com/product_dashboard/acoustic_modems. [Accessed: 02 September 2018].

[42] "Desert Star Systems: sam-1_technical_reference." [Online]. Available: http://www.desertstar.com/page/sam-1. [Accessed: 02 September 2018].

[43] "DSPComm - Connecting Everything Underwater." [Online]. Available: https://dspcommgen2.com/aquacomm-gen2-next-generation-acoustic-modem/. [Accessed: 02 September 2018].

[44] "Underwater Communication Systems | develogic." [Online]. Available: http://www.develogic.de/products/underwater-communication-systems/. [Accessed: 02 September 2018].

[45] "uComm Underwater Acoustic Modem." [Online]. Available: https://www.sonardyne.com/product/underwater-acoustic-modems/. [Accessed: 02 September 2018].

\section{AUTHORS}

Dr. Faiza Al-Salti received her B.Sc., M.Sc. and PhD Degrees in computer science from the Sultan Qaboos University (Oman) in 2012, 2015 and 2019, respectively. She is currently an assistant professor in Muscat College (Oman). Her research interest includes communication protocols, terrestrial and underwater wireless sensor networks, and Internet of Things.

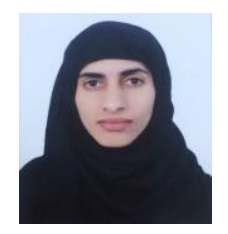

Dr. Nasser Alzeidi received his $\mathrm{PhD}$ degree in Computer Science from the University of Glasgow (UK) in 2007. He is currently an assistant professor of computer science and the director of the Center for Information Systems at Sultan Qaboos University, Oman. His research interests include performance evaluation of communication systems, wireless networks, interconnection networks, System on Chip architectures and parallel and distributed computing. He is a member of the

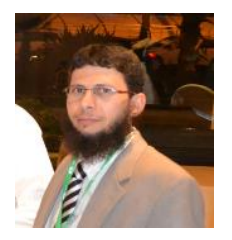
IEEE. 